\title{
A Large Oral Melanoma: A Case Report of a Rare but Aggressive Malignancy
}

\author{
${ }^{1}$ Division of Oral and Maxillofacial Surgery, Faculty of Dentistry, \\ Thammasat University, Pathum Thani, Thailand \\ 2Department of Oral Biomedical Sciences, Research Group of \\ Chronic Inflammatory Oral Diseases and Systemic Diseases \\ Associated with Oral Health, Faculty of Dentistry, Khon Kaen \\ University, Khon Kaen, Thailand \\ ${ }^{3}$ Oral and Maxillofacial Surgery Clinic, Udon Thani Hospital, \\ Udon Thani, Thailand
}

Kritsasith Warin ${ }^{1, \odot}$ Poramaporn Klanrit ${ }^{2}$ Nutdanai Pattanajakr ${ }^{3}$

\begin{abstract}
Address for correspondence Kritsasith Warin, DDS, FRCDT, Division of Oral and Maxillofacial Surgery, Faculty of Dentistry, Thammasat University, Pathum Thani 12121, Thailand (e-mail: warin@tu.ac.th).
\end{abstract}

Eur J Dent 2021;15:812-816.

\begin{abstract}
A variety of black-pigmented lesions of the oral cavity can be found, ranging from harmless benign lesions such as melanotic macule, smoker's melanosis, amalgam/graphite tattoos, and pigmented nevus to a life-threatening oral malignant melanoma. Oral melanoma is a rare and aggressive malignant tumor that originates from melanocytes' proliferation and accounts for only $0.5 \%$ of all oral malignancies. The etiology is unknown. Most oral melanomas are present at the palate and the upper

Keywords

- melanoma

- oral cavity

- pathology

- immunohistochemistry

- palliative care alveolar ridge, whereas occurrences at the buccal mucosa, the lower alveolar ridge, and the lip are rare, with only a few reports in the literature. The diagnosis is confirmed by a biopsy. The prognosis is poor, with a 5-year survival rate of $\sim 20 \%$. In this report, we present a case of large oral melanoma at the right buccal mucosa involving the right lower alveolar ridge and lip commissure, which are relatively unusual locations for oral melanoma. In addition, immunohistochemical markers used for diagnostic, therapeutic, and prognostic decision-making of oral melanoma are also discussed.
\end{abstract}

\section{Introduction}

Melanoma is a malignant neoplasm comprising abnormal melanocytes, which originates from neural crest cells. Oral melanoma is a rare neoplasm that represents $\sim 2 \%$ of all melanomas and $0.5 \%$ of all oral malignancies, occurring much less frequently than its cutaneous relatives. ${ }^{1-3}$ Most oral melanoma studies report a wide range of ages and are more common in males than females. ${ }^{4,5}$ The most regular site of occurrence is the palate, followed by the upper alveolar ridge. Other oral sites are the lower alveolar ridge, tongue, buccal mucosa, upper and lower lip. $2.5,6$

Varied clinical features of oral melanoma have been reported. The most common presentation is an asymptomatic brown, dark blue, or black macule, sometimes with erythema or ulceration. ${ }^{1}$ Histologically, melanomas comprise medium-to-large cells that may be polyhedral, fusiform, round, spindle, pleomorphic, epithelioid, microcytic, or various shapes. ${ }^{7}$ Immunohistochemically, melanomas variously express S-100 protein and melanocytic markers, including HMB-45, melanoma antigen recognized by T-cells 1 , or melanoma antigen A (MART-1/Melan-A), tyrosinase, and microphthalmia-associated transcription factor (MITF), ${ }^{3,7}$ The prognosis for patients with oral melanoma is poor. These patients' poor outcomes may be partly a result of a delay in diagnosis. ${ }^{8}$

This article presented a case of large oral melanoma at the right buccal mucosa involving the right lower alveolar ridge

\section{(C) 2021. European Journal of Dentistry.}

This is an open access article published by Thieme under the terms of the Creative Commons Attribution-NonDerivative-NonCommercial-License, permitting copying and reproduction so long as the original work is given appropriate credit. Contents may not be used for commercial purposes, or adapted, remixed, transformed or built upon. (https://creativecommons.org/licenses/by-nc-nd/4.0/).

Thieme Medical and Scientific Publishers Pvt. Ltd. A-12, 2nd Floor, Sector 2, Noida-201301 UP, India 
and lip commissure in a 69-year-old female who preferred to obtain palliative care rather than surgery or other alternative treatments, including radiotherapy, chemotherapy, and immunotherapy.

\section{Case Report}

A 69-year-old Thai female patient presented to the oral and maxillofacial clinic to evaluate an asymptomatic black lesion at her right buccal mucosa. The patient reported that the lesion had been present for 4 months with a gradual increase in size. Extraoral examination revealed no cervical lymphadenopathy and no facial paresthesia. Intraoral examination showed asymptomatic scattered black patches, exhibiting flat, irregular, nodular, and ulcerative surfaces, at the right buccal mucosa extending posteriorly to the right alveolar ridge and anteriorly to the lip commissure. The size of the lesion was $\sim 5 \times 3 \mathrm{~cm}$ (-Fig. 1). An incisional biopsy was performed at the buccal mucosal lesion, which showed a prominent nodular surface, and a computed tomography (CT) scan was taken.

The CT scan revealed a heterogeneous, enhancing mass with central hypodensity involving the right lower alveolar ridge with the destruction of the right mandible and bulging in a subcutaneous, right buccal region. The lesion's total size was $\sim 4.7 \times 2.4 \times 1.9 \mathrm{~cm}$ (-Fig. 2). Histopathological findings demonstrated several atypical, pleomorphic melanocytes

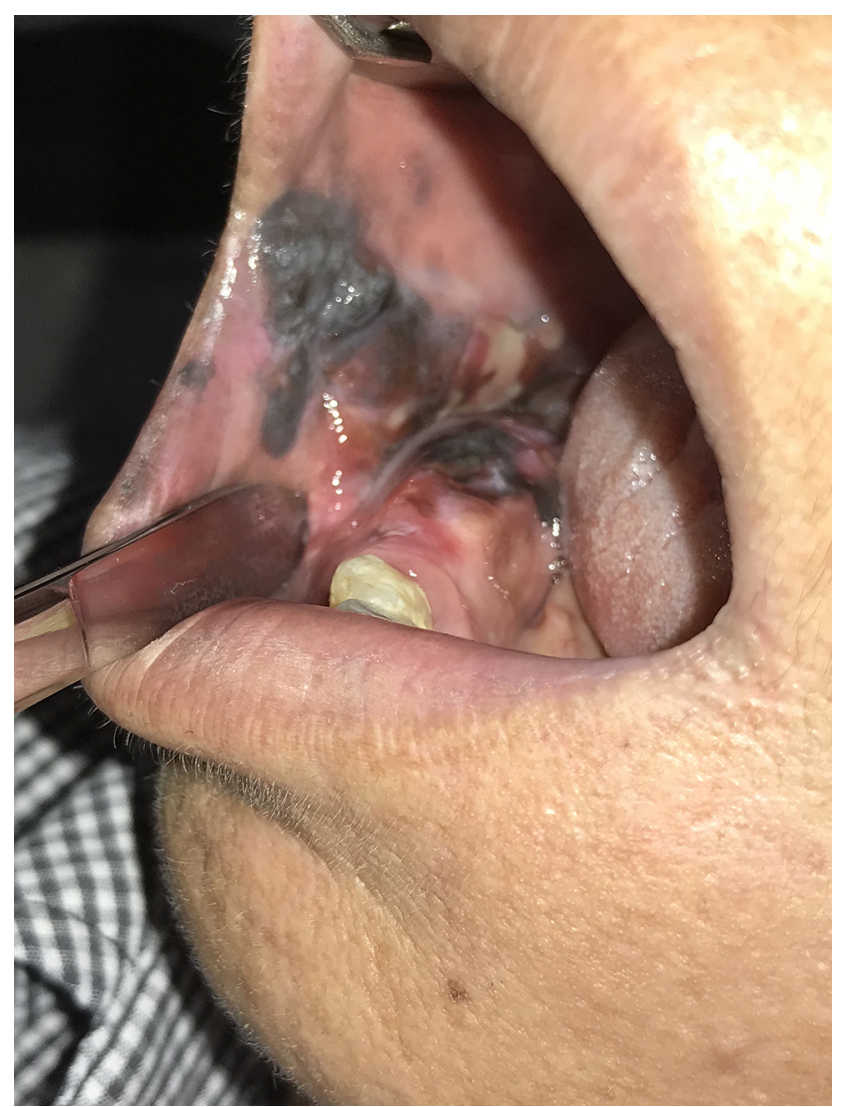

Fig. 1 Clinical appearance. Asymptomatic scattered black patches exhibiting flat, irregular, nodular, and ulcerative surfaces, at the right buccal mucosa extending posteriorly to the right alveolar ridge and anteriorly to the lip commissure. The size of the lesion was $\sim 5 \times 3 \mathrm{~cm}$.

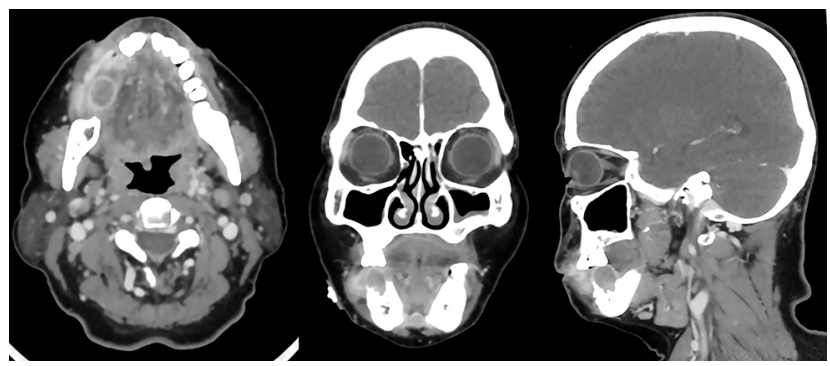

Fig. 2 Computed tomography scan revealed a heterogeneous, enhancing mass with central hypodensity involving the right lower alveolar ridge with the destruction of the right mandible and bulging in a subcutaneous, right buccal region. The total size of the lesion was $\sim 4.7 \times 2.4 \times 1.9 \mathrm{~cm}$.

with melanin pigments in basal and some suprabasal cell layers of the epithelium. These pleomorphic cells are strongly positive for S-100, HMB-45, and Melan-A. An early invasion of atypical melanocytes containing melanin pigments into the superficial lamina propria was seen. The pathological diagnosis of oral melanoma with a superficial invasion was confirmed (-Fig. 3). Another panel of immunohistochemical staining further demonstrated the expression of BRAF (V600E) and CD117 (c-kit) of atypical cells at the superficially invasive and in situ components (-Fig. 4).

Although the pathological diagnosis of an incisional biopsy from the right buccal mucosa showed a superficial invasion of oral melanoma, the bone invasion of the lesion at the right mandible demonstrated by a CT scan indicated an advanced stage of oral melanoma. On the follow-up day, we informed the patient about the diagnosis and treatment options, including surgical treatment and/or chemoradiotherapy. She denied radical surgery and preferred alternative medical treatment. Therefore, she was referred to be treated by the oncologist for radiation therapy and/or systemic chemotherapy. Later, with the pandemic of coronavirus disease 2019 (COVID-19), the patient subsequently chose palliative care, in which some of the appointments were delivered by telemedicine instead of previously planned radiation therapy with chemotherapy. She received morphine for analgesia, metoclopramide for antiemetic, and diazepam or lorazepam for adjuvant therapy. After 4 months of follow-up, she continued to refuse radiotherapy and systemic chemotherapy. Her caregiver informed us that the patient was satisfied with this mode of treatment.

\section{Discussion}

Oral melanoma is a rare neoplasm and very aggressive tumor of melanocytic origin, accounting for only $0.5 \%$ of all oral malignancies. It frequently involves the hard palate and upper alveolar ridge but rarely occurs in the buccal mucosa, lower alveolar ridge, and lip., ${ }^{2,6}$ The pathogenesis of oral mucosal melanomas has not yet confirmed whether mucosal melanocytes' dysfunction is the main cause. Most studies have reported that the lesion occurs more frequently in males than females, with an average age of 53.,6 The exact etiology of oral melanoma is unknown. However, smoking 


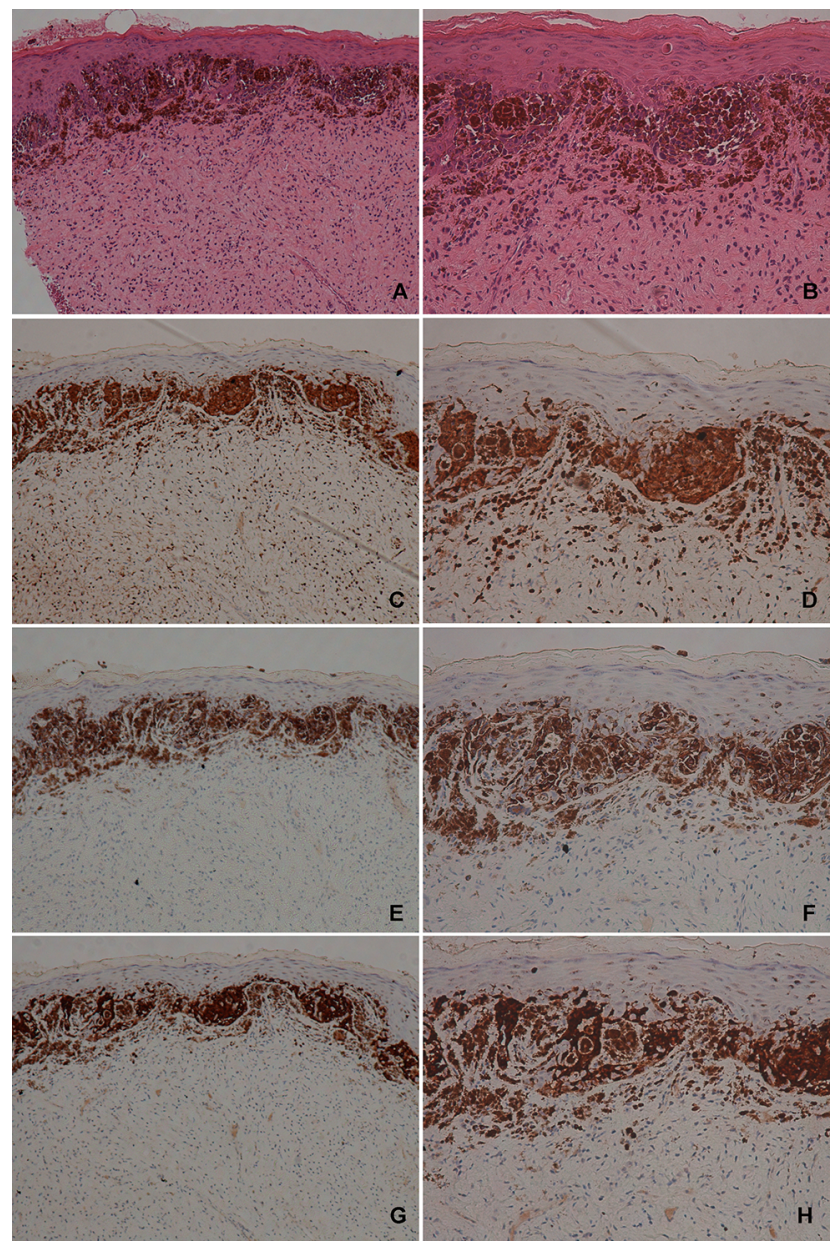

Fig. 3 Histopathologic features. (A, B) Hematoxylin and eosin stained section demonstrated several atypical, pleomorphic melanocytes with melanin pigments in basal and some suprabasal cell layers of the epithelium. A pagetoid spread of some melanocytes to the superficial layer of epithelium is observed $(\mathbf{A}: \times 100, \mathbf{B}: \times 200)$. (C, D) S-100 (C: $\times 100$, D: $\times 200) .(E, F)$ HMB-45 (E: $\times 100, F: \times 200) .(G, H)$ Melan-A (G: $\times 100, \mathbf{H}: \times 200)$.

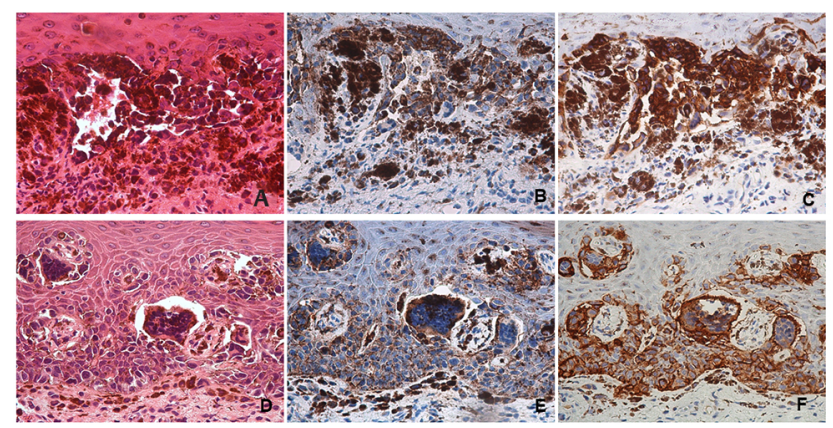

Fig. 4 The hematoxylin and eosin-stained section and immunohistochemical study of BRAF (V600E) and CD117 (c-kit) at the superficially invasive area $(\mathbf{A}-\mathbf{C})$ and in situ component of an incisional biopsy specimen (D-F). (A, D) Hematoxylin and eosin ( $\times 400)$. (B, E) BRAF (V600E) (×400). (C, F) CD117 (c-kit) (×400).

and chronic irritation caused by ill-fitting dentures, as well as swallowing and inhaling environmental carcinogens at elevated internal body temperature, have been proposed to play a role in the development of malignant lesions., ${ }^{3,9}$
Clinically, oral melanoma demonstrates as a slow-growing, asymptomatic, brown, purple, or black pigmentation with irregular borders and may appear as a rapidly expanding mass with or without ulceration. ${ }^{1,10}$ The clinical differential diagnosis should exclude oral melanoma from normal variations ${ }^{11}$ and other benign, harmless black lesions such as melanotic macule, smoker's melanosis, amalgam and graphite tattoos, and pigmented nevus. ${ }^{12}$ In this case report, we encountered a large oral melanoma at the buccal mucosa, involving the lower alveolar ridge and lip commissure. A CT scan highlighted an invasion of a lesion into the right mandible. According to previous studies, oral melanomas of the buccal mucosa, lower alveolar ridge, and lip, which are infrequent locations, have been found in only 4 to $7 \%$ of all oral melanomas. ${ }^{2,4}$ Another learning point of this case was that an incisional biopsy's pathological result revealed only a superficial invasion of oral melanoma. Even though we carefully selected the darkest and the most raised region of the lesion at the buccal mucosa for the incisional biopsy, this area was not representative of the true infiltrative and destructive nature of this lesion as represented by a CT scan that showed a large destructive area in the right mandible. However, clinical features consisting of the "ABCD" warning signs (A: asymmetry; $\mathrm{B}$ : border irregularities; $\mathrm{C}$ : color variegation; D: diameter $>6 \mathrm{~mm})^{3}$ and radiographic presentations of this case, in conjunction with histopathological features, offered a diagnosis of oral melanoma.

The histopathology of oral malignant melanoma is similar to cutaneous melanoma, with an initial phase characterized by a "radial growth phase" followed by an invasion of the underlying tissues known as the "vertical growth phase." The malignant tumor cells of oral malignant melanoma exhibit a wide variety of forms, including spindle cells, plasmacytoid cells, clear cells, small round blue cells, and epithelioid cells, organized into sheets, organoid/alveolar, or desmoplastic formation. ${ }^{13}$ The general histopathologic appearance of this case demonstrated atypical melanocytes with melanin pigments, horizontally spreading in the basal and suprabasal cell layers, along with a superficial invasion of these atypical cells into the superficial lamina propria.

Immunohistochemical study, positive staining for S-100 protein, MART-1 or Melan-A, tyrosinase, and HMB-45 strongly confirm the diagnosis of melanoma. ${ }^{2,3}$ In this case, the pathological diagnosis was confirmed by the expression of S- 100 protein, Melan-A, and HMB- 45 by tumor cells, as shown in - Fig. 3. Besides, it has been suggested that MITF serves as a useful immunohistochemical marker in diagnosing melanoma. Furthermore, studies show that MITF has high sensitivity and specificity for metastatic melanoma, and positivity has been reported in S-100 negative melanoma, ${ }^{14,15}$ indicating that MITF may be another optional immunohistochemical marker to confirm the diagnosis of oral melanoma. The mutational analysis for BRAF and KIT mutations, which are primarily found in an advanced stage of melanoma, is another beneficial molecular test for therapeutic decision making for the most appropriate targeted therapy in treating melanoma to improve patient survival rate. ${ }^{16}$ In this case, we did not perform the mutational 
analysis of BRAF and KIT but found that BRAF (V600E) and CD117 (c-kit) protein aberrantly expressed in our case by an immunohistochemical study (-Fig. 4).

The 5-year survival rate for patients with oral melanoma remains poor and has been reported to be 13 to $38 \%{ }^{17,18}$ The poor outcome of these patients may be, in part, the result of a delay in diagnosis. Widespread metastases are the leading cause of death in patients with oral melanoma. Oral melanoma metastases develop in regional lymph nodes or distant organs such as the brain and breast. ${ }^{19-21}$ Since the mucosal surfaces of the oral cavity are not regularly self-examined, and these surfaces are not visible to others, the lesion may remain asymptomatic and continue to grow overlooked for some period of time.

The treatment of oral melanoma is currently controversial, and there is no consensus on the best therapeutic approach. However, surgery remains the main modality of treatment, with additional radiotherapy and/or chemotherapy to prevent recurrence and metastasis. Although patients have received aggressive resection and multimodal treatments, the prognosis of oral melanoma is still inferior. ${ }^{18,22}$ Palliative treatment, including symptomatic management with painkillers such as morphine and other adjuvant medicines such as metoclopramide or benzodiazepine, is also an alternative option for cancer management. ${ }^{23}$

In this case, the patient finally chose symptomatic palliative treatment, requiring a regular stay at or travel to the hospital. Unfortunately, there was an outbreak of COVID-19, a fatal disease, especially for cancer patients. ${ }^{24}$ Therefore, some of the present case's appointments were provided via telemedicine. As an alternative option to hospitalization, telemedicine has played an essential role in oral cancer palliative care in the COVID-19 era. Symptomatic medication adjustments and palliative care counseling can be provided to the caregivers and patients to prevent the spread of COVID-19 infection. ${ }^{25}$

Based on our experience in managing patients with large oral melanoma, there are some key issues to consider. Clinicians should be aware of the dreadfulness of oral melanoma and rule it out from other benign black pigmented lesions with similar clinical findings, as the behavior and survival rate of these two types of tumors are different. Also, clinicians should provide sufficient information on how harmful this lesion is to patients, direct patients to get prompt diagnosis and appropriate early treatment, and avoid a delay in diagnosis, which can significantly reduce patients' survival rates.

\section{Conclusion}

This case report highlighted the rare occurrence of an extensive oral melanoma at the buccal mucosa, involving the lower alveolar ridge and lip commissure. Biologically, this type of malignancy shows aggressive behavior and poor survival. Like this case, most previously reported cases reveal a delay in diagnosis, leading to widespread lesions and swelling. Due to the poor prognosis of this disease, precise early diagnosis of these tumors can help clinicians plan appropriate treatment to prevent tumor extension and reduce the risk of metastasis, leading to the improved prognosis of patients.

\section{Ethical Approval}

This study was ethically approved by the institutional ethics committee because of its retrospective nature. All the procedures performed were part of the routine care.

\section{Funding \\ None.}

\section{Conflict of Interest}

None declared.

\section{References}

1 Femiano F, Lanza A, Buonaiuto C. Gombos F, Di Spirito F, Cirillo N. Oral malignant melanoma: a review of the literature. J Oral Pathol Med 2008;37(7):383-388

2 Hicks MJ, Flaitz CM. Oral mucosal melanoma: epidemiology and pathobiology. Oral Oncol 2000;36(2):152-169

3 Meleti M, Leemans CR, Mooi WJ. Vescovi P, van der Waal I. Oral malignant melanoma: a review of the literature. Oral Oncol 2007;43(2):116-121

4 Shen ZY, Liu W, Bao ZX, Zhou ZT, Wang LZ. Oral melanotic macule and primary oral malignant melanoma: epidemiology, location involved, and clinical implications. Oral Surg Oral Med Oral Pathol Oral Radiol Endod 2011;112(1):e21-e25

5 Smith MH, Bhattacharyya I, Cohen DM, et al. Melanoma of the oral cavity: an analysis of 46 new cases with emphasis on clinical and histopathologic characteristics. Head Neck Pathol 2016;10(3):298-305

6 Sortino-Rachou AM, Cancela MdeC, Voti L, Curado MP. Primary oral melanoma: population-based incidence. Oral Oncol 2009;45(3):254-258

7 López F, Rodrigo JP, Cardesa A, et al. Update on primary head and neck mucosal melanoma. Head Neck 2016;38(1):147-155

8 Rapidis AD, Apostolidis C, Vilos G, Valsamis S. Primary malignant melanoma of the oral mucosa. J Oral Maxillofac Surg 2003;61(10):1132-1139

9 Prasetyaningtyas N, Jatiatmaja NA, Radithia D, et al. The response of the tongue epithelial on cigarette smoke exposure as a risk factor for oral cancer development. Eur J Dent 2021;15(2):320-324

10 Francisco AL, Furlan MV, Peresi PM, et al. Head and neck mucosal melanoma: clinicopathological analysis of 51 cases treated in a single cancer centre and review of the literature. Int J Oral Maxillofac Surg 2016;45(2):135-140

11 Surboyo MDC, Ernawati DS, Parmadiati AE, Marsetyo RI. Pigmented fungiform papillae of the tongue and lingual fimbriae as single presentation in adult: a case report and literature review. Eur J Dent 2020;14(4):702-706

12 Alawi F. Pigmented lesions of the oral cavity: an update. Dent Clin North Am 2013;57(4):699-710

13 Umeda M, Shimada K. Primary malignant melanoma of the oral cavity-its histological classification and treatment. $\mathrm{Br}$ J Oral Maxillofac Surg 1994;32(1):39-47

14 Miettinen M, Fernandez M, Franssila K, Gatalica Z, Lasota J, Sarlomo-Rikala M. Microphthalmia transcription factor in the immunohistochemical diagnosis of metastatic melanoma: comparison with four other melanoma markers. Am J Surg Pathol 2001;25(2):205-211

15 Ohsie SJ, Sarantopoulos GP, Cochran AJ, Binder SW. Immunohistochemical characteristics of melanoma. J Cutan Pathol 2008;35(5):433-444

16 Pincet L, Lambercy K, Pasche P, Broome M, Latifyan S, Reinhard A. Mucosal melanoma of the head and neck: 
a retrospective review and current opinion. Front Surg 2021;7:616174

17 Chatzistefanou I, Kolokythas A, Vahtsevanos K, Antoniades K. Primary mucosal melanoma of the oral cavity: current therapy and future directions. Oral Surg Oral Med Oral Pathol Oral Radiol 2016;122(1):17-27

18 Sun CZ, Chen YF, Jiang YE, Hu ZD, Yang AK, Song M. Treatment and prognosis of oral mucosal melanoma. Oral Oncol 2012;48(7):647-652

19 Alqahtani S, Alhefdhi AY, Almalik O, et al. Primary oral malignant melanoma metastasis to the brain and breast: a case report and literature review. Oncol Lett 2017;14(2):1275-1280

20 Keller DS, Thomay AA, Gaughan J, et al. Outcomes in patients with mucosal melanomas. J Surg Oncol 2013;108(8):516-520
21 Zbytek B, Carlson JA, Granese J. Ross J, Mihm MC Jr, Slominski A. Current concepts of metastasis in melanoma. Expert Rev Dermatol 2008;3(5):569-585

22 Umeda M, Komatsubara H, Shigeta T, et al. Treatment and prognosis of malignant melanoma of the oral cavity: preoperative surgical procedure increases risk of distant metastasis. Oral Surg Oral Med Oral Pathol Oral Radiol Endod 2008;106(1):51-57

23 Cleary J. Essential medicines in palliative care. Palliat Med 2014;28(4):291-292

24 Liang W, Guan W, Chen R, et al. Cancer patients in SARS-CoV-2 infection: a nationwide analysis in China. Lancet Oncol 2020;21(3):335-337

25 Mehta AK, Smith TJ. Palliative care for patients with cancer in the COVID-19 era. JAMA Oncol 2020;6(10):1527-1528 